\title{
VARIABILITY IN A YOUNG, L/T TRANSITION PLANETARY-MASS OBJECT ${ }^{*}$
}

\author{
Beth A. Biller ${ }^{1,2}$, Johanna Vos ${ }^{1}$, Mariangela Bonavita ${ }^{1}$, Esther Buenzli ${ }^{2}$, Claire Baxter ${ }^{1}$, Ian J. M. Crossfield ${ }^{3}$, \\ Katelyn Allers ${ }^{4}$, Michael C. Liu ${ }^{5}$, Mickaël Bonnefoy ${ }^{6,7}$, Niall Deacon ${ }^{8}$, Wolfgang Brandner ${ }^{2}$, \\ Joshua E. Schlieder ${ }^{9,15}$, Trent Dupuy ${ }^{10}$, Taisiya Kopytova ${ }^{2,11}$, Elena Manjavacas ${ }^{12}$, France Allard ${ }^{13}$, \\ DereK HOMEIER ${ }^{14}$, AND ThOMAS HenNing ${ }^{2}$ \\ ${ }^{1}$ Institute for Astronomy, University of Edinburgh, Blackford Hill View, Edinburgh EH9 3HJ, UK \\ ${ }^{2}$ Max-Planck-Institut für Astronomie, Königstuhl 17, D-69117 Heidelberg, Germany \\ ${ }^{3}$ Lunar \& Planetary Laboratory, University of Arizona, 1629 E. University Boulevard, Tucson, AZ 95721, USA \\ ${ }^{4}$ Department of Physics and Astronomy, Bucknell University, Lewisburg, PA 17837, USA \\ ${ }^{5}$ Institute for Astronomy, University of Hawaii, 2680 Woodlawn Drive, Honolulu, HI 96822, USA \\ ${ }^{6}$ University of Grenoble Alpes, IPAG, F-38000 Grenoble, France \\ ${ }^{7}$ CNRS, IPAG, F-38000 Grenoble, France \\ ${ }^{8}$ Centre for Astrophysics Research, University of Hertfordshire, College Lane, Hatfield AL10 9AB, UK \\ ${ }^{9}$ NASA Ames Research Center, Moffett Field, CA, USA \\ ${ }^{10}$ Department of Astronomy, University of Texas, 2515 Speedway C1400, Austin, TX 78712, USA \\ ${ }^{11}$ International Max-Planck Research School for Astronomy and Cosmic Physics at the University of Heidelberg, IMPRS-HD, Heidelberg, Germany \\ ${ }^{12}$ Instituto de Astrofsica de Canarias (IAC), C/ Vía Láctea s/n, E-38205, La Laguna, Tenerife, Spain \\ ${ }^{13}$ CRAL-ENS, 46, Allée d'Italie, F-69364 Lyon Cedex 07, France \\ ${ }^{14}$ Zentrum für Astronomie der Universität Heidelberg, Landessternwarte, Königstuhl 12, D-69117 Heidelberg, Germany \\ Received 2015 August 20; accepted 2015 October 8; published 2015 October 30
}

\begin{abstract}
As part of our ongoing NTT SoFI survey for variability in young free-floating planets and low-mass brown dwarfs, we detect significant variability in the young, free-floating planetary-mass object PSO J318.5-22, likely due to rotational modulation of inhomogeneous cloud cover. A member of the $23 \pm 3$ Myr $\beta$ Pic moving group, PSO $\mathrm{J} 318.5-22$ has $T_{\text {eff }}=1160_{-40}^{+30} \mathrm{~K}$ and a mass estimate of $8.3 \pm 0.5 M_{\text {Jup }}$ for a $23 \pm 3$ Myr age. PSO J318.5-22 is intermediate in mass between 51 Eri $\mathrm{b}$ and $\beta$ Pic $\mathrm{b}$, the two known exoplanet companions in the $\beta$ Pic moving group. With variability amplitudes from $7 \%$ to $10 \%$ in $J_{S}$ at two separate epochs over $3-5$ hr observations, we constrain the rotational period of this object to $>5 \mathrm{hr}$. In $K_{S}$, we marginally detect a variability trend of up to $3 \%$ over a $3 \mathrm{hr}$ observation. This is the first detection of weather on an extrasolar planetary-mass object. Among L dwarfs surveyed at high photometric precision $(<3 \%)$, this is the highest amplitude variability detection. Given the low surface gravity of this object, the high amplitude preliminarily suggests that such objects may be more variable than their high-mass counterparts, although observations of a larger sample are necessary to confirm this. Measuring similar variability for directly imaged planetary companions is possible with instruments such as SPHERE and GPI and will provide important constraints on formation. Measuring variability at multiple wavelengths can help constrain cloud structure.
\end{abstract}

Key words: brown dwarfs - planets and satellites: atmospheres - planets and satellites: gaseous planets

\section{INTRODUCTION}

Of the current ensemble of $\sim 30$ free-floating young planetary-mass objects (Gagné et al. 2014, 2015), PSO J318.5-22 (Liu et al. 2013) is the closest analog in properties to imaged exoplanet companions. Gagné et al. (2014) and Liu et al. (2013) identify it as a $\beta$ Pic moving group member (23 \pm 3 Myr; Mamajek \& Bell 2014) and it possesses colors and magnitudes similar to the HR 8799 planets (Marois et al. 2008, 2010) and 2M1207-39b (Chauvin et al. 2005). PSO $\mathrm{J} 318.5-22$ has $T_{\text {eff }}=1160_{-40}^{+30} \mathrm{~K}$ and a published mass estimate of 6.5 $5_{-1.0}^{+1.3} M_{\text {Jup }}$ for an age of $12 \mathrm{Myr}$ (Liu et al. 2013), rising to $8.3 \pm 0.5 M_{\text {Jup }}$ for the updated age of $23 \pm 3 \mathrm{Myr}$ (Allers et al. 2015). PSO J318.5-22 is intermediate in mass and luminosity between 51 Eri b ( $2 M_{\text {Jup }}$; Macintosh et al. 2015) and $\beta$ Pic b ( 11-12 $M_{\text {Jup }}$; Lagrange et al. 2010; Bonnefoy et al. 2014), the two known exoplanet companions in the $\beta$ Pic moving group. Because PSO J318.5-22 is free floating, it enables highprecision characterization not currently possible for exoplanet

\footnotetext{
* Based on observations made with ESO Telescopes at the La Silla Paranal Observatory under program ID 095.C-0590.

15 NASA Postdoctoral Program Fellow.
}

companions to bright stars. In particular, we report here the first detection of photometric variability in a young, $\mathrm{L} / \mathrm{T}$ transition planetary-mass object.

Variability is common for cool brown dwarfs, but until now has not been probed for lower-mass planetary objects with similar effective temperatures. Recent large-scale surveys of brown dwarf variability with Spitzer have revealed mid-IR variability of up to a few percent in $>50 \%$ of L- and T-type brown dwarfs (Metchev et al. 2015). Buenzli et al. (2014) find that $\sim 30 \%$ of the L5-T6 objects surveyed in their HST SNAP survey show variability trends, and large ground-based surveys also find widespread variability (Radigan 2014; Radigan et al. 2014; Wilson et al. 2014). While variability amplitude may be increased across the $\mathrm{L} / \mathrm{T}$ transition (Radigan et al. 2014), variability is now robustly observed across a wide range of $\mathrm{L}$ and $\mathrm{T}$ spectral types. We therefore expect variability in young extrasolar planets, which share similar $T_{\text {eff }}$ and spectral types but lower surface gravity. In fact, Metchev et al. (2015) tentatively find a correlation between low surface gravity and high amplitude variability in their L dwarf sample.

Observed field brown dwarf variability is likely produced by rotational modulation of inhomogeneous cloud cover over the 
Table 1

SOFI Observations of PSO J318.5-22

\begin{tabular}{lccccc}
\hline \hline Date & Filter & DIT & NDIT & $\begin{array}{c}\text { Total Expo- } \\
\text { sure Time }\end{array}$ & $\begin{array}{c}\text { On- } \\
\text { sky Time }\end{array}$ \\
\hline 2014 Oct 9 & $J_{S}$ & $10 \mathrm{~s}$ & 6 & $3.80 \mathrm{hr}$ & $5.15 \mathrm{hr}$ \\
2014 Nov 9 & $J_{S}$ & $15 \mathrm{~s}$ & 6 & $2.40 \mathrm{hr}$ & $2.83 \mathrm{hr}$ \\
2014 Nov 10 & $K_{S}$ & $20 \mathrm{~s}$ & 6 & $2.80 \mathrm{hr}$ & $3.16 \mathrm{hr}$
\end{tabular}

3-12 hr rotational periods of these objects (Zapatero Osorio et al. 2006). Apai et al. (2013) and Buenzli et al. (2015) find that their variability amplitude as a function of wavelength are best fit by a combination of thin and thick cloud layers. We expect a similar mechanism to drive variability in planetarymass objects with similar $T_{\text {eff }}$, albeit with potentially longer periods, as these objects will not yet have spun up with age. Only a handful of directly imaged exoplanet companions are amenable to variability searches using high-contrast imagers such as SPHERE at the VLT (Beuzit et al. 2008) and GPI at Gemini (Macintosh et al. 2014); to search for variability in a larger sample of planetary-mass objects and young, very low mass brown dwarfs, we have been conducting the first survey for free-floating planet variability using NTT SoFI (Moorwood et al. 1998). We have observed 22 objects to date, of which 7 have mass estimates $<13 M_{\text {Jup }}$, and all have mass estimates $<25 M_{\text {Jup }}$. PSO J318.5-22 is the first variability detection from this survey.

\section{OBSERVATIONS AND DATA REDUCTION}

We obtained three data sets for PSO J318.5-22 with NTT SoFI (0!" 288/pixel, 4!92 × 4!.92 field of view) in 2014 October and November. Observations are presented in Table 1. We attempted to cover as much of the unknown rotation period as possible; however, scheduling constraints and weather conditions limited our observations to $2-5 \mathrm{hr}$ on sky. In search mode, we observed in $J_{S}$; however, we did obtain a $K_{S}$ follow-up light curve for PSO J318.5-22. We nodded the target between two positions on the chip, ensuring that at each jump from position to position, the object is accurately placed on the same original pixel. This allowed for sky-subtraction, while preserving photometric stability. We followed an ABBA nodding pattern, taking three exposures at each nod position.

Data were corrected for crosstalk artifacts between quadrants, flat-fielded using special dome flats that correct for the "shade" (illumination dependent bias) found in SoFI images, and illumination-corrected using observations of a standard star. Sky frames for each nod position were created by median combining normalized frames from the other nod positions closest in time. These were then re-scaled to and subtracted from the science frame. Aperture photometry for all sources on the frame were acquired using the IDL task aper.pro with aperture radii of $4,4.5,5,5.5,6$, and 6.5 pixels and background subtraction annuli from 21 to 31 pixels.

\section{LIGHT CURVES}

We present the final binned $J_{S}$ light curve from 2014 October (with detrended reference stars for comparison) in Figure 1 and the final binned $J_{S}$ and $K_{S}$ light curves from 2014 November in Figure 2. Raw light curves obtained from aperture photometry display fluctuations in brightness due to

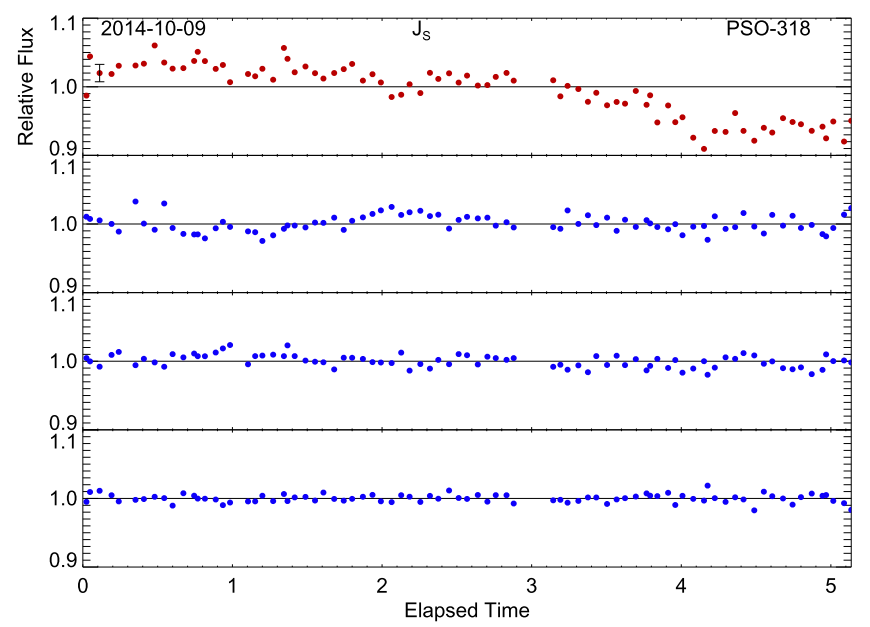

Figure 1. Final binned $J_{S}$ light curve and comparison detrended reference stars from 2014 October 9. Typical error bars are shown on the first photometric point. The variability amplitude at this epoch is $>10 \%$ with a period of $>5 \mathrm{hr}$.
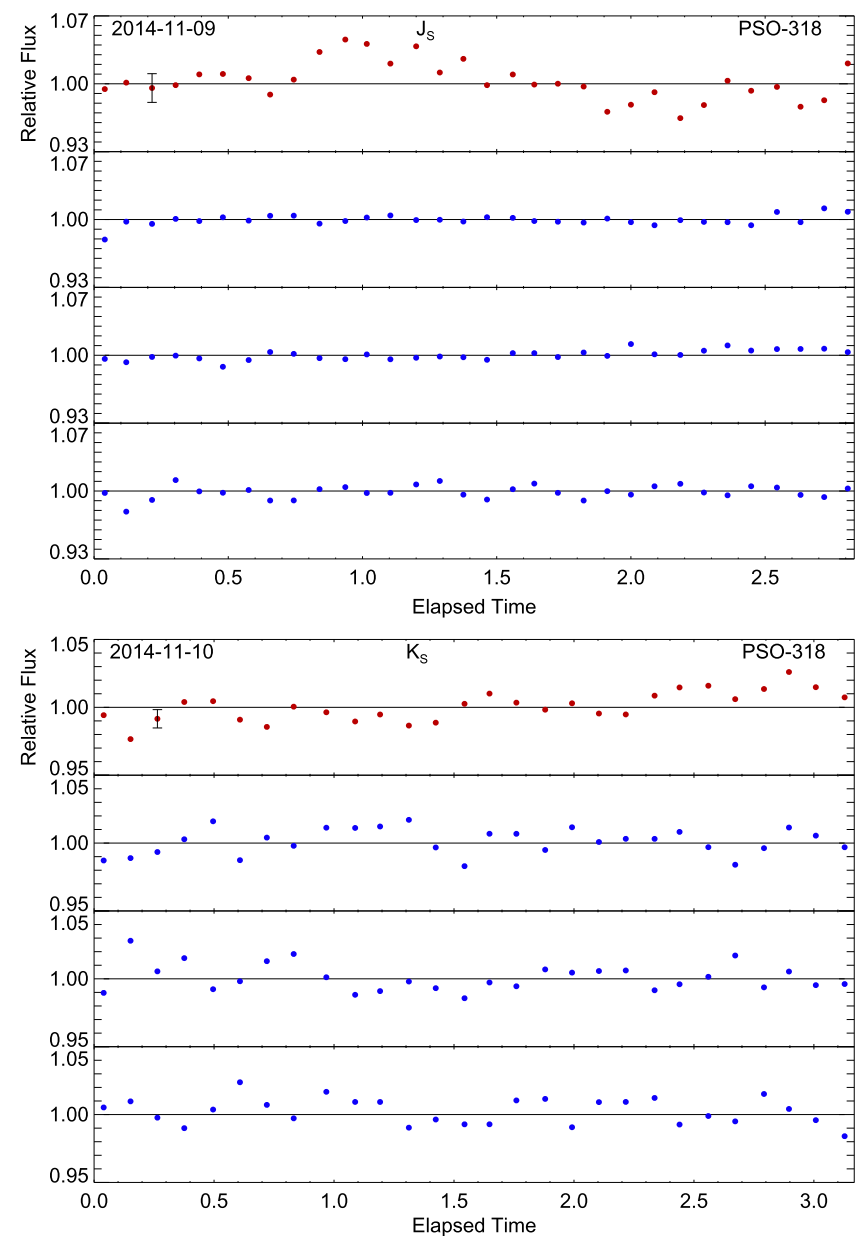

Figure 2. Top: final binned $J_{S}$ light curve from 2014 November 9. Bottom: final binned $K_{S}$ light curve from 2014 November 11. Light curves are presented similarly as in Figure 1. The $J_{S}$ variability amplitude at this epoch is $>7 \%$ with a period of $\geqslant 3 \mathrm{hr}$. We marginally detect $K_{S}$ variability, with amplitude up to $3 \%$ over our $3 \mathrm{hr}$ observation.

changing atmospheric transparency, airmass, and residual instrumental effects. These changes can be removed via division of a calibration curve calculated from carefully chosen, well-behaved reference stars (Radigan et al. 2014). 
To detrend our light curves, first we discarded potential reference stars with peak flux values below 10 or greater than $10,000 \mathrm{ADU}$ (where array nonlinearity is limited to $<1.5 \%$ ). Different nods were normalized via division by their median flux before being combined to give a relative flux light curve. For each star a calibration curve was created by median combining all other reference stars (excluding that of the target and star in question). The standard deviation and linear slope for each light curve were calculated, and stars with a standard deviation or slope $\sim 1.5-3$ times greater than that of the target were discarded. This process was iterated until a set of well-behaved reference stars was chosen. Final detrended light curves were obtained by dividing the raw curve for each star by its calibration curve. The best light curves shown here are with the aperture that minimizes the standard deviation after removing a smooth polynomial (as done in Biller et al. 2013)_for all epochs, the 4 pixel aperture (similar to the point-spread function FWHM) yielded the best result. Final light curves are shown binned by a factor of threecombining all three exposures taken in each ABBA nod position. Error bars were calculated in a similar manner as in Biller et al. (2013) - a low-order polynomial was fit to the final light curve and then subtracted to remove any astrophysical variability and the standard deviation of the subtracted light curve was adopted as the typical error on a given photometric point (shown in each light curve as the error bar given on the first photometric point). As a check, we also measured photometry and light curves using both the publicly available aperture photometry pipeline from Radigan (2014) as well as the psf-fitting pipeline described in Biller et al. (2013). Results from all three pipelines were consistent.

We found the highest amplitude of variability in our $J_{S}$ light curve from 2014 October 9-over the five hours observed, PSO J318.5-22 varies by $10 \pm 1.3 \%$. The observed variability does not correlate with airmass changes-the target was overhead for the majority of this observation, with airmass between 1 and 1.2 for the first $3 \mathrm{hr}$, increasing to $\sim 2$ by the end of the observation. The flattening of the light curve from 4 to $5 \mathrm{hr}$ elapsed time in our light curve may be indicative of a minimum in the light curve. However, as no clear repetition of maxima or minima have been covered, the strongest constraints we can place on the rotational period and variability amplitude for PSO J318.5-22 in this epoch is that the period must be $>5 \mathrm{hr}$ and the amplitude must be $\geqslant 10 \%$. If the variation is sinusoidal, these observations point to an even longer period of $>7-8 \mathrm{hr}$.

On 2014 November 9 , we recovered $J_{S}$ variability with a somewhat smaller amplitude of $7 \pm 1 \%$ over our three-hourlong observation. A maximum is seen $1 \mathrm{hr}$ into the observation, and a potential minimum is seen at $2 \mathrm{hr}$ into the observation. The observed variability is not correlated with airmass changes during the observation-the observation started at airmass $=1.1$, increasing steadily to airmass $=2.0$ at the end of the observation. If the variability is roughly sinusoidal and single peaked, this observation would suggest a period of $\sim 3 \mathrm{hr}$; however, we cannot constrain the period beyond requiring it to be $>3 \mathrm{hr}$, as we have not covered multiple extrema and as the light curve could potentially be double peaked (Radigan et al. 2012). The light curve evolved considerably between the 2014 October and November epochs - a phenomena also found in other older variable brown dwarfs (Artigau et al. 2009; Radigan et al. 2012, 2014; Gillon et al. 2013; Metchev et al. 2015).

On 2014 November 11, we obtained a $K_{S}$ light curve for PSO J318.5-22. Given its extremely red colors, PSO J318.5-22 is brighter in $K_{S}$ than $J_{S}$ and is one of the brightest objects in the SoFI field. Thus, we attain higher photometric precision in our $K_{S}(0.7 \%)$ light curve compared to $J_{S}(1 \%-$ $1.3 \%$ ). Fitting slopes to the target and three similarly bright reference stars, the target increases in flux by $0.9 \%$ per hour, while the reference stars have slopes of $0.1 \%-0.6 \% / \mathrm{hr}$ (consistent with a flat line within our photometric precision). Thus, we tentatively find a marginal variability trend of up to $3 \%$ over our $3 \mathrm{hr}$ observation, requiring reobservation to be confirmed. Additionally, in this case the tentative variability is not completely uncorrelated with airmass changes - during this observation, airmass increased steadily from 1.1 to 2.2.

\section{DISCUSSION}

This is the first detection of variability in such a cool, low surface gravity object. While variability has been detected previously for very young $(<1-2 \mathrm{Myr})$ planetary-mass objects in star-forming regions such as Orion (cf. Joergens et al. 2013), such variability is driven by a different mechanism than expected for PSO J318.5-22. These previous detections have been for $\mathrm{M}$ spectral type objects with much higher $T_{\text {eff }}$ than PSO J318.5-22. At these temperatures, variability is driven by starspots induced by the magnetic fields of these objects or ongoing accretion. PSO J318.5-22 is too cool to have starspots and likely too old for ongoing accretion. From its red colors, PSO J318.5-22 must be entirely cloudy (Liu et al. 2013). Thus, the likely mechanism producing the observed variability is inhomogeneous cloud cover, as has been found previously to drive variability in higher-mass brown dwarfs with similar $T_{\text {eff }}$ (Artigau et al. 2009; Radigan et al. 2012, 2014; Apai et al. 2013; Buenzli et al. 2014, 2015; Radigan 2014; Wilson et al. 2014). Notably, among L dwarfs surveyed at high photometric precision $(<3 \%)$, PSO J318.5-22's J-band variability amplitude is the highest measured for an L dwarf to date (cf. Yang et al. 2015; Buenzli et al. 2015)_reinforcing the suggestion by Metchev et al. (2015) that variability amplitudes might be typically larger for lower-gravity objects.

To model cloud-driven as well as hotspot variability, we follow the approach of Artigau et al. (2009) and Radigan et al. (2012), combining multiple $1 \mathrm{~d}$ models to represent different regions of cloud cover. We consider the observed atmosphere of our object to be composed of flux from two distinct cloud regions (varying in temperature and/or in cloud prescription) with fluxes of $F_{1}$ and $F_{2}$, respectively, and with a minimum filling fraction for the $F_{2}$ region of $a$. The peak-to-trough amplitude of variability ( $\Delta F / F$, i.e., the change of flux divided by the mid-brightness flux) observed in a given bandpass due a change of filling fraction over the course of the observation is given by Equation (2) from Radigan et al. (2012), where $\Delta a$ is the change in filling factor over the observation, $\Delta F=F_{2}-$ $F_{1}$, and $\alpha=a+0.5 \Delta a$, the filling fraction of the $F_{2}$ regions at 

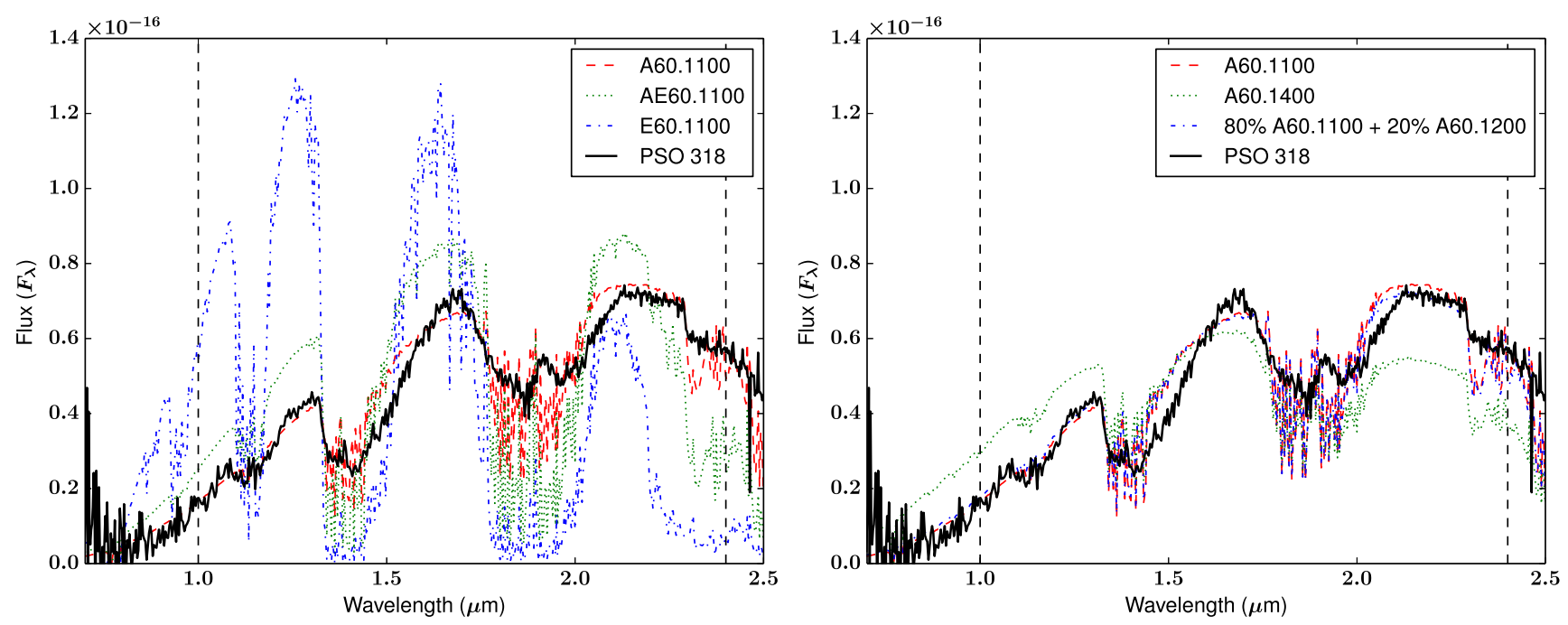

Figure 3. Left: best-fit single-model spectra for model A, AE, and E clouds from Madhusudhan et al. (2011) overlaid on the spectrum of PSO J318.5-22 presented in Liu et al. (2013). The overall best-fit model has $T_{\text {eff }}=1100 \mathrm{~K}, \log (g)=4$, solar metallicity, and model A (thick) clouds. AE and E cloud models fail to reproduce the observed spectrum. Right: $T_{\text {eff }}=1400 \mathrm{~K}, \log (g)=4$, solar metallicity, and model A (thick) cloud spectrum as well as the best-fit multi-component spectrum, consisting of $80 \% T_{\text {eff }}=1100 \mathrm{~K}+20 \% T_{\text {eff }}=1200 \mathrm{~K}$ model A clouds overplotted on the Liu et al. (2013) spectrum. Hotter models do not fit the observed features of the Liu et al. (2013) spectrum; the combined $1100 \mathrm{~K}+1200 \mathrm{~K}$ model spectrum reproduces the observed spectrum marginally better than the best-fit single-component model.

mid-brightness:

$$
\begin{aligned}
& \left((1-a-\Delta a) F_{1}\right. \\
A= & \left.+(a+\Delta a) F_{2}-(1-a) F_{1}-a F_{2}\right) / \\
& \left(0 . 5 \left[(1-a-\Delta a) F_{1}\right.\right. \\
& \left.\left.+(a+\Delta a) F_{2}+(1-a) F_{1}+a F_{2}\right]\right) \\
= & \frac{\Delta a}{\alpha+F_{1} / \Delta F} .
\end{aligned}
$$

We calculated the synthetic photon fluxes $F_{1}$ and $F_{2}$ using the cloudy exoplanet models of Madhusudhan et al. (2011) and the filter transmissions provided for the SoFI $J_{S}$ and $K_{S}$ filters. While a diversity of brown dwarf/exoplanet cloud models are available (e.g., Allard et al. 2001, 2012; Saumon \& Marley 2008), the Madhusudhan et al. (2011) models are particularly tuned to fit the cloudy atmospheres and extremely red colors of young, low surface gravity objects such as the HR 8799 exoplanets (Marois et al. 2008, 2010). As PSO J318.5-22 is a free-floating analog of these exoplanets, the Madhusudhan et al. (2011) models are the optimal choice for this analysis. Because PSO J318.5-22's extraordinarily red colors preclude clear patches in its atmosphere (Liu et al. 2013), we consider only combinations of cloudy models. The Madhusudhan et al. (2011) models model the cloud distribution according to a shape function, $f(P)$ :

$$
f(P)= \begin{cases}\left(P / P_{\mathrm{u}}\right)^{s_{\mathrm{u}}} & P \leqslant P_{\mathrm{u}} \\ f_{\text {cloud }} & P_{\mathrm{u}} \leqslant P \leqslant P_{\mathrm{d}} \\ \left(P / P_{\mathrm{d}}\right)^{-s_{\mathrm{d}}} & P \geqslant P_{\mathrm{d}},\end{cases}
$$

where $P_{\mathrm{u}}$ and $P_{\mathrm{d}}$ are the pressures at the upper and lower pressure cutoffs of the cloud and $P_{\mathrm{u}}<P_{\mathrm{d}}$. The indices $s_{\mathrm{u}}$ and $s_{\mathrm{d}}$ control how rapidly the clouds dissipate at their upper and lower boundaries. We consider combinations of three cloud models from Madhusudhan et al. (2011), with $60 \mu \mathrm{m}$ grain sizes and solar metallicity:

$$
\begin{aligned}
& \text { Model E: } s_{\mathrm{u}}=6, s_{\mathrm{d}}=10, f_{\text {cloud }}=1 \\
& \text { Model A: } s_{\mathrm{u}}=0, s_{\mathrm{d}}=10, f_{\text {cloud }}=1 \\
& \text { Model AE: } s_{\mathrm{u}}=1, s_{\mathrm{d}}=10, f_{\text {cloud }}=1
\end{aligned}
$$

where model E cuts off rapidly at altitude; model A provides the thickest clouds, extending all the way to the top of the atmosphere; and model AE provides an intermediate case.

Fitting single-component models to the spectrum presented in Liu et al. (2013), we find that the best single-component fit is for model A prescription clouds with $T_{\text {eff }}=1100 \mathrm{~K}$ (see Figure 3). This agrees well with the derived $T_{\text {eff }}=1100_{-40}^{+30} \mathrm{~K}$ from Liu et al. (2013). We thus adopt $T_{\text {eff }}=1100 \mathrm{~K}$ as the temperature of the dominant cloud component, with a second cloud component at $T_{2}$. Explicitly fitting multi-cloud component models, we find that a combination of $80 \%$ model A clouds with $T_{\text {eff }}=1100 \mathrm{~K}$ and $20 \%$ model A clouds with $T_{\text {eff }}=1200 \mathrm{~K}$ marginally fit the spectrum better than a singlecomponent fit. Multi-component fits using multiple cloud prescriptions do not fit the spectrum well-model A clouds (or similar) are likely the dominant cloud component in this atmosphere. We did not attempt further analysis of the spectrum in terms of variable cloud components, as the spectrum was observed at a different epoch than the variability monitoring.

We then calculated synthetic fluxes in $J_{S}$ and $K_{S}$ for models with all three cloud prescriptions, $T_{\text {eff }}$ from $700-1700 \mathrm{~K}$, and $\log (g)=4$ (matching the measured $\log (g)$ of PSO J318.5-22 from Liu et al. 2013). Then, considering different values for $a$, we solved for $\Delta a$ from Equation (1) for the maximum observed amplitude in $J_{S}$, with $T_{1}=1000 \mathrm{~K}$, different values of $T_{2}$, and varying cloud prescriptions (plotted in the bottom panels of Figure 4 for a minimum $T_{2}$ filling fraction of 0.2 ). Filling fraction significantly varies for small $\Delta T$, but only small variations in filling factor can drive variability for abs $(\Delta T)>200 \mathrm{~K}$. Considering different values for $a$, we calculated the variability amplitude ratio $A_{K_{S}} / A_{J_{S}}$ for the same 

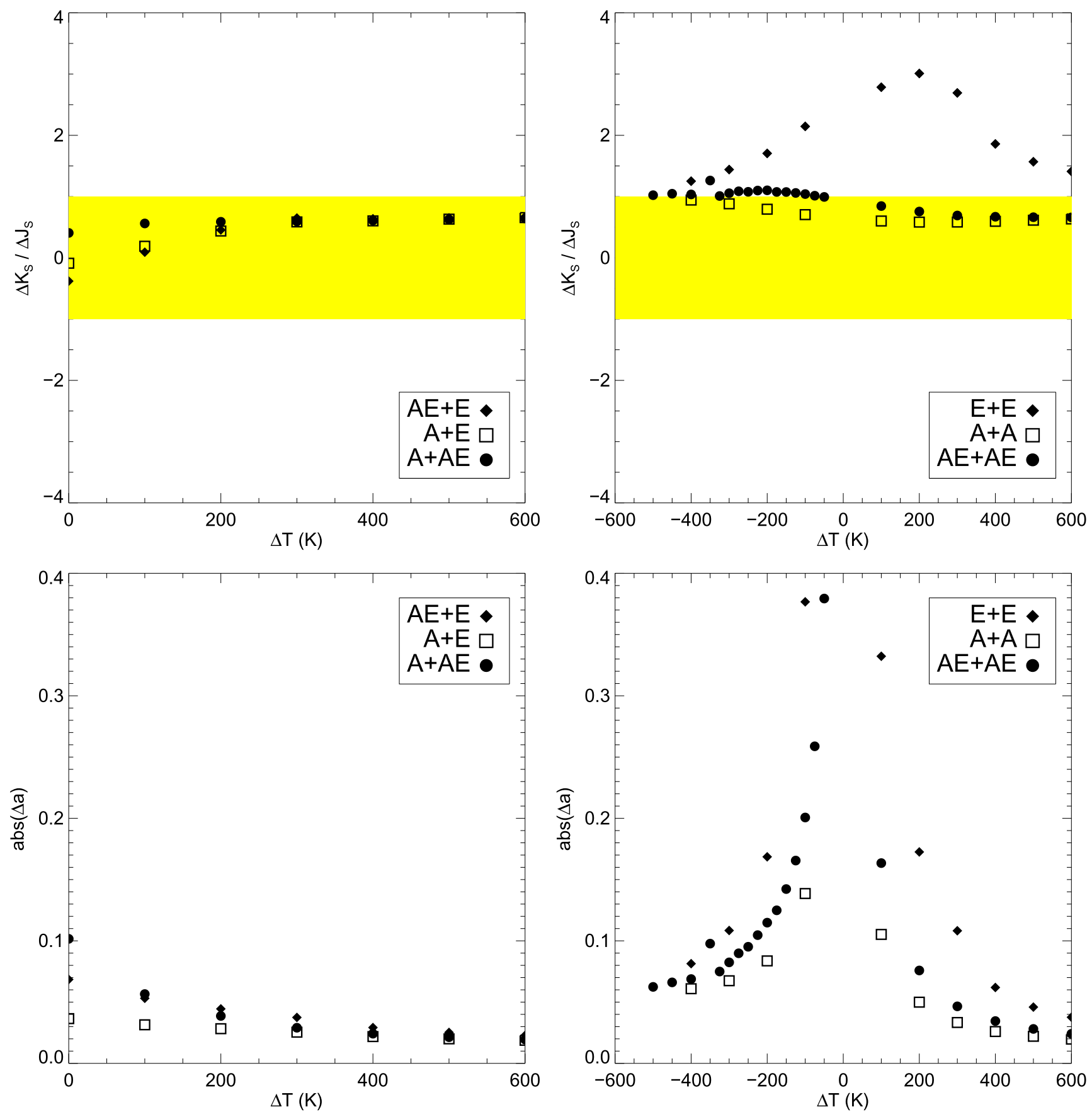

Figure 4. Top left and top right: predicted $K_{S}$ to $J_{S}$ amplitude ratio $A_{K_{S}} / A_{J_{S}}$ as a function of $\Delta T$, the temperature difference between cloud components at $T_{1}$ and $T_{2}$, and for a filling fraction of the $T_{2}$ regions of 0.2. Inhomogeneous cloud cover is plotted on the left (AE+E, A+E, A+AE) and homogeneous cloud cover is plotted on the right $(\mathrm{E}+\mathrm{E}, \mathrm{A}+\mathrm{A}, \mathrm{AE}+\mathrm{AE})$. The yellow region denotes the values of the amplitude ratio that have previously been found for variable field brown dwarfs. Bottom left and bottom right: maximum change in filling fraction needed to produce the observed amplitude $A_{J_{S}}$ as a function of $\Delta T$.

combinations of $T_{1}, T_{2}$, and varying cloud prescriptions. We adopt the same convention as Radigan et al. (2012), where the thicker cloud prescription is used for the $F_{1}$ regions. In the inhomogeneous cloud case, we also assume that the thinner cloud producing the $\mathrm{F}_{2}$ region is at a hotter $T_{\text {eff }}$ than the $\mathrm{F}_{1}$ regions (i.e., the thin cloud top is deeper in the atmosphere and thus hotter), so $\Delta T=T_{2}-T_{1}>0$. Representative results for predicted amplitude ratio are presented in Figure 4-similar to Radigan et al. (2012), different minimum filling fractions yield qualitatively similar results, so we present only $a=0.2$ results here. Inhomogeneous combinations of clouds are shown on the left, homogeneous combinations on the right (i.e., hotspots instead of cloud patchiness as the driver of variability).

Observations of variable brown dwarfs have generally found $\operatorname{abs}\left(A_{K_{S}} / A_{J_{S}}\right)<1$ (see, e.g., Artigau et al. 2009; Radigan et al. 2012, 2014; Radigan 2014; Wilson et al. 2014); thus, we shade this region in yellow in Figure 4. As we have not yet covered a whole period of this variability nor do we have simultaneous multi-wavelength observations, we cannot determine $A_{K_{S}} / A_{J_{S}}$ with the data in hand. It remains to be seen whether abs $\left(A_{K_{S}} / A_{J_{S}}\right)$ is also $<1$ for PSO J318.5-22, which is much redder in $J-K$ than the high- $g$, bluer objects for which $A_{K_{S}} / A_{J_{S}}$ is 
robustly measured. Future observations that cover the entire period of variability at multiple wavelengths are necessary to characterize the source of this variability. However, in advance of these observations, it is instructive to consider what amplitude ratios can be produced for young, low surface gravity objects with thick clouds.

In the case of inhomogeneous cloud cover $(\mathrm{E}+\mathrm{AE}, \mathrm{E}+\mathrm{A}, \mathrm{A}$ $+\mathrm{AE})$, combinations of thick clouds can produce $A_{K_{S}} / A_{J_{S}}<1$, for $\Delta T>150$, similar to what was found by Radigan et al. (2012) for the field early T 2MASS J21392676+0220226. However, while Radigan et al. (2012) found that singlecomponent cloud models from Saumon \& Marley (2008) with $f_{\text {sed }}=3$ always have $A_{K_{S}} / A_{J_{S}}>1$, we do not find this to be the case with all of the Madhusudhan et al. (2011) cloud models. This is true in the $\mathrm{E}+\mathrm{E}$ case, but for combinations of thicker cloud models $(\mathrm{AE}+\mathrm{AE}, \mathrm{A}+\mathrm{A}), A_{K_{S}} / A_{J_{S}}$ can be $<1$. Unlike Radigan et al. (2012), who rule out homogeneous cloud cover with hotspots as a source of variability for the T1.5 brown dwarf 2MASS J21392676+0220226 based on a measured $A_{K_{S}} / A_{J_{S}}<1$, a measurement of $A_{K_{S}} / A_{J_{S}}<1$ for young, low surface gravity objects with thick clouds would be consistent with both inhomogeneous clouds (patchy cloud cover) and homogeneous clouds (hotspots).

\section{CONCLUSIONS}

We detect significant variability in the young, free-floating planetary-mass object PSO J318.5-22, suggesting that planetary companions to stars with similar colors (e.g., the HR 8799 planets) may also be variable. With variability amplitudes from $7 \%$ to $10 \%$ in $J_{S}$ at two separate epochs over $3-5 \mathrm{hr}$ observations, we constrain the period to $>5 \mathrm{hr}$, likely $>7-8 \mathrm{hr}$ in the case of sinusoidal variation. In $K_{S}$, we marginally detect a variability trend of up to $3 \%$ over our $3 \mathrm{hr}$ observation. Our marginal detection suggests that the variability amplitude in $K_{S}$ may be smaller than that in $J_{S}$, but simultaneous multi-wavelength observations are necessary to confirm this. Using the models of Madhusudhan et al. (2011), combinations of both homogeneous and inhomogeneous cloud prescriptions can tentatively model variability with abs $\left(A_{K_{S}} / A_{J_{S}}\right)<1$ for young, low surface gravity objects with thick clouds.

Only one exoplanet rotation period has been measured to date-7-9 hr for $\beta$ Pic b (Snellen et al. 2014). PSO J318.5-22 is only the second young planetary-mass object with constraints placed on its rotational period and is likely also a fast rotator like $\beta$ Pic $\mathrm{b}$, with possible rotation periods from $\sim 5$ to $20 \mathrm{hr}$. PSO J318.5-22 is thus an important link between the rotational properties of exoplanet companions and those of old, isolated Y dwarfs with similar masses.

E.B. was supported by the Swiss National Science Foundation (SNSF). We thank the anonymous referee for useful comments that helped improve the manuscript. This work was supported by a consolidated grant from STFC. D.H. acknowledges support from the European Research Council under the European Community's Seventh Framework Programme (FP7/2007-2013 Grant Agreement No. 247060) and from the Collaborative Research Centre SFB 881 "The Milky Way System" (subproject A4) of the German Research Foundation (DFG).

\section{REFERENCES}

Allard, F., Hauschildt, P. H., Alexander, D. R., Tamanai, A., \& Schweitzer, A. 2001, ApJ, 556, 357

Allard, F., Homeier, D., \& Freytag, B. 2012, RSPTA, 370, 2765

Allers, K. N., Gallimore, J. F., Liu, M. C., \& Dupuy, T. J. 2015, ApJ, submitted Apai, D., Radigan, J., Buenzli, E., et al. 2013, ApJ, 768, 121

Artigau, É., Bouchard, S., Doyon, R., \& Lafrenière, D. 2009, ApJ, 701, 1534 Beuzit, J.-L., Feldt, M., Dohlen, K., et al. 2008, Proc. SPIE, 7014, 701418 Biller, B. A., Crossfield, I. J. M., Mancini, L., et al. 2013, ApJL, 778, L10 Bonnefoy, M., Marleau, G.-D., Galicher, R., et al. 2014, A\&A, 567, L9 Buenzli, E., Apai, D., Radigan, J., Reid, I. N., \& Flateau, D. 2014, ApJ, 782, 77

Buenzli, E., Marley, M. S., Apai, D., et al. 2015, ApJ, 812, 163

Buenzli, E., Saumon, D., Marley, M. S., et al. 2015, ApJ, 798, 127

Chauvin, G., Lagrange, A.-M., Dumas, C., et al. 2005, A\&A, 438, L25

Gagné, J., Faherty, J. K., Cruz, K. L., et al. 2015, arXiv:1506.07712

Gagné, J., Lafrenière, D., Doyon, R., Malo, L., \& Artigau, É 2014, ApJ, 783,121

Gillon, M., Triaud, A. H. M. J., Jehin, E., et al. 2013, A\&A, 555, L5

Joergens, V., Bonnefoy, M., Liu, Y., et al. 2013, A\&A, 558, L7

Lagrange, A.-M., Bonnefoy, M., Chauvin, G., et al. 2010, Sci, 329, 57

Liu, M. C., Magnier, E. A., Deacon, N. R., et al. 2013, ApJL, 777, L20

Macintosh, B., Graham, J. R., Barman, T., et al. 2015, arXiv:1508.03084

Macintosh, B., Graham, J. R., Ingraham, P., et al. 2014, PNAS, 111, 12661

Madhusudhan, N., Burrows, A., \& Currie, T. 2011, ApJ, 737, 34

Mamajek, E. E., \& Bell, C. P. M. 2014, MNRAS, 445, 2169

Marois, C., Macintosh, B., Barman, T., et al. 2008, Sci, 322, 1348

Marois, C., Zuckerman, B., Konopacky, Q. M., Macintosh, B., \& Barman, T. 2010, Natur, 468, 1080

Metchev, S. A., Heinze, A., Apai, D., et al. 2015, ApJ, 799, 154

Moorwood, A., Cuby, J.-G., \& Lidman, C. 1998, Msngr, 91, 9

Radigan, J. 2014, ApJ, 797, 120

Radigan, J., Jayawardhana, R., Lafrenière, D., et al. 2012, ApJ, 750, 105

Radigan, J., Lafrenière, D., Jayawardhana, R., \& Artigau, E. 2014, ApJ, 793,75

Saumon, D., \& Marley, M. S. 2008, ApJ, 689, 1327

Snellen, I. A. G., Brandl, B. R., de Kok, R. J., et al. 2014, Natur, 509, 63

Wilson, P. A., Rajan, A., \& Patience, J. 2014, A\&A, 566, A111

Yang, H., Apai, D., Marley, M. S., et al. 2015, ApJL, 798, L13

Zapatero Osorio, M. R., Martín, E. L., Bouy, H., et al. 2006, ApJ, 647, 1405 